\title{
Letter to the editor: authors' response
}

Dear editors

We thank Drs Webb and Bryon for their comments $^{1}$ on our TIDES paper. ${ }^{2}$ We are responding to their critiques of our study. Beyond clarifying the 'devilish details' they highlighted, we want to emphasise the clinical significance of our results ${ }^{2}$ - a substantial proportion of our patients reported elevated symptoms of depression (17\%) and anxiety (29\%) across nine countries. Higher rates were reported by parents: depression $337.3 \%$ of mothers, $31.3 \%$ of fathers) and anxiety $(47.8 \%$ of mothers, $35.2 \%$ of fathers). Although the 'absolute' number of individuals who have depression or anxiety is difficult to ascertain with any screening tool, it is clear that psychological distress is common and has been associated with worse: self-management, pulmonary 
function, quality of life, and increased hospitalisations and healthcare costs. ${ }^{34}$ We can no longer minimise the extent of the problem and its impact on the health of individuals with $\mathrm{CF}$.

Our response to the 'details': First, 6088 participants completed the depression and anxiety screening, with a small minority $(n=63,1 \%)$ failing to provide a birth date or age and were excluded from analyses stratified by age (sample $=6025)$. Second, data from the two screening measures were pooled because each was the 'gold standard' at the time in Europe and USA; participating countries were given the choice. The historical argument in favour of the HADS-D has been its removal of somatic items for those with a chronic illness, but our data on the CES-D showed that none of the three most commonly endorsed symptoms related to physical disease. Recent articles also faulted the HADS for its insensitivity, unstable factor structure and lack of correspondence with diagnostic criteria. ${ }^{5}$ Thus, rates of depression on the HADS are likely an underest $i$ mate, and consistent with our results, which found more positive screens on the CES-D versus HADS-D. Third, a conservative strategy was used; a 'case' was identified as an elevation on one or the other measure, essentially 'imputing' a zero for measures not administered. We reported estimates for both measures, but used the combined definition in subsequent analyses and discussion.

TIDES was a 'grassroots' effort growing out of concerns voiced by CF teams in Europe and the USA that patients and parents were experiencing psychological symptoms not systematically identified or treated. Oxley and Webb published a commentary reporting that $63 \%$ of referrals to the clinical psychologist at the Manchester Adult CF Centre were for depression and anxiety and called for annual screening of psychopathology. ${ }^{6}$

The editorial challenged us to state how this study is groundbreaking. Let us count the ways. First, these results are robust because they were drawn from the largest sample ever screened in a rare disease across Europe and the USA, increasing their ecological validity and generalisability. Second, no study of this size and cultural diversity has measured depressive and anxious symptoms in parents caring for children with a serious, chronic disease. We uncovered rates that were both concerning and a call to action. Third, we analysed paired data in over 1100 parent-teen dyads and found an increased probability of psychological distress in adolescents whose parents screened positive, highlighting the link between parent-child mental health. Finally, our results challenge the long-held belief that somatic symptoms (eg, fatigue) drive the commonly reported elevations in depression for individuals with chronic illness and inflate depression scores. Comparisons of the HADS-D and CES-D showed these symptoms were not the most frequently reported. Our inclusion of two screening instruments illustrated the need for standardisation of mental health assessment. If we can agree on a screening measure, this variable can be added to our respective registries and estimate a more accurate point prevalence, evaluate what precedes an elevated screen (eg, drop in lung function) and identify health outcomes that follow an elevation (eg, worse adherence). A recent survey of CF providers across Europe and the USA indicated that at least 48 different screening measures of psychological distress are in use. ${ }^{7}$ Thus, standardisation of this assessment process is needed.

TIDES led to an International Guidelines Committee on Mental Health in CF sponsored by the CF Foundation and ECFS. This multinational, multidisciplinary group of providers and researchers examined recent data on screening measures and set a high bar: the measures had to be free, evidence excellent sensitivity/ specificity, available in all major languages, and converge with diagnostic criteria. We were not wedded to a particular screening tool. Most important was gathering the evidence-which has led to an innovative, international effort to systematically measure and treat psychological distress. And yes, it is both groundbreaking and an enormous leap forward!

\section{Alexandra L Quittner, ${ }^{1}$ Dave Barker, ${ }^{2}$ Lutz Goldbeck, ${ }^{3}$ Alistair Duff, ${ }^{4}$ Janice Abbott $^{5}$}

${ }^{1}$ Department of Psychology \& Pediatrics, University of Miami, Coral Gables, Florida, USA

${ }^{2}$ Department of Psychiatry and Human Behavior, Brown University, Providence, Rhode Island, USA

${ }^{3}$ Department of Child and Adolescent Psychiatry/ Psychotherapy, University Hospital Ulm, Ulm, Germany ${ }^{4}$ The Regional Paediatric CF Unit, Leeds Teaching Hospitals NHS Trust, Leeds, UK
${ }^{5}$ School of Psychology, University of Central Lancashire, Preston, UK

Correspondence to Dr Alexandra L Quittner, Department of Psychology \& Pediatrics, University of Miami, 5665 Ponce de Leon Blvd, Coral Gables, FL 33146, USA; aquittner@miami.edu

Contributors ALQ is the lead author, grant-holder, manager of database, data collection, data analysis and interpreter of the original TIDES paper and lead author of this response. DB contributed to the response regarding data analyses. $L G, A D$ and $J A$ assisted in reviewing and editing the response. Final version was approved by all authors.

Funding This work was supported by cystic fibrosis foundations in seven countries.

Competing interests None.

Provenance and peer review Not commissioned; internally peer reviewed.

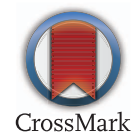

To cite Quittner AL, Barker D, Goldbeck L, et al. Thorax 2015;70:490-491.

Received 17 December 2014

Revised 18 December 2014

Accepted 9 January 2015

Published Online First 30 January 2015

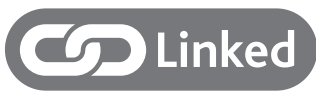

http://dx.doi.org/10.1136/thoraxjnl-2015-206787

Thorax 2015;70:490-491.

doi:10.1136/thoraxjnl-2014-206708

\section{REFERENCES}

1 Webb AK, Bryon M. The International Depression Epidemiological Study (TIDES): unfinished business? Thorax 2014;69:1067-8.

2 Quittner AL, Goldbeck L, Abbott J, et al. Prevalence of depression and anxiety in patients with cystic fibrosis and parent caregivers: results of The International Depression Epidemiological Study across nine countries. Thorax 2014;69:1090-7.

3 Smith BA, Modi AC, Quittner AL, et al. Depressive symptoms in children with cystic fibrosis and parents and its effects on adherence to airway clearance. Pediatr Pulmonol 2010;45:756-63.

4 Snell C, Fernandes S, Bujoreanu IS, et al. Depression, illness severity, and healthcare utilization in cystic fibrosis. Pediatr Pulmonol 2014;49:1177-81.

5 Cameron IM, Cardy A, Crawford JR, et al. Measuring depression severity in general practice: discriminatory performance of the PHQ-9, HADS-D, and BDI-II. Br J Gen Pract 2011;61:e419-26.

6 Oxley $H$, Webb AK. How a clinical psychologist manages the problems of adults with cystic fibrosis. J Roy Soc Med 2005;98(Suppl 45):37-46.

7 Abbott J, Elborn S, Georgiopoulos A, et al. Cystic Fibrosis Foundation and European Cystic Fibrosis Society survey of cystic fibrosis mental health care delivery. J Cyst Fibros. Published Online: 12 Jan 2015 doi:10.1016/j.jcf.2014.12.015. 